> Les cellules possèdent des réseaux protéiques permettant de percevoir des stimulus externes et de réagir à ceux-ci. Ces réseaux sont décomposables en voies de signalisation qui transmettent les signaux du microenvironnement à divers effecteurs cellulaires. Les études sur la signalisation par les récepteurs à activité tyrosine kinase (RTK) ont été les premières à décrire le mécanisme par lequel un signal extracellulaire est transmis jusqu'au noyau pour induire une réponse transcriptionnelle. Quoique moins bien connues, les études génétiques utilisant la drosophile ou le nématode ont contribué de manière déterminante à façonner notre compréhension de la signalisation par les RTK. Nous décrivons ici brièvement l'apport de ces organismes à la mise en évidence des nombreuses protéines qui forment ou modulent l'axe de signalisation RTK/RAS/ERK. <

Les récepteurs à activité tyrosine kinase (RTK) jouent un rôle prépondérant dans divers processus cellulaires de base, comme la régulation de la prolifération, de la différenciation et de la survie cellulaires. L'action des RTK passe principalement par l'activation de voies de signalisation effectrices, dont l'une des mieux caractérisées, et probablement des plus influentes, est communément appelée la voie RAS/ERK (extracellular signal-regulated kinase). La description des événements moléculaires reliant l'activation des RTK à la modulation de facteurs de transcription par la kinase ERK (également connue sous le nom de MAPK, mitogen-activated protein kinase) a constitué l'une des percées majeures en signalisation cellulaire. Au milieu des années 1980, les premiers liens étaient déjà tissés par des données reliant l'activité de la petite GTPase RAS, I'oncoprotéine la plus fréquemment mutée dans les cancers humains [1], à celle d'une autre oncoprotéine bien connue, le RTK EGFR (epidermal growth factor receptor) [2]. Ces études initiales ont donné lieu à une série de découvertes qui ont permis de comprendre comment un signal extracellulaire est transmis à l'intérieur de la cellule. Ici, nous nous foca-

\section{La signalisation RTK/RAS/ERK élargie}

\section{Contributions}

de la génétique

à l'assemblage d'un réseau de signalisation

Dariel Ashton-Beaucage, Marc Therrien

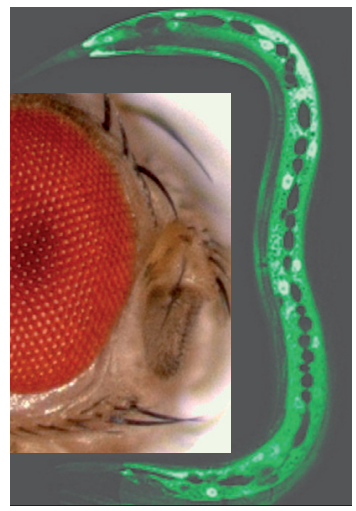

D. Ashton-Beaucage :

Institut de recherche en immunologie et cancérologie, Université de Montréal, CP 6128, succursale centre-ville, Montréal, Québec, Canada.

M. Therrien : Institut

de recherche en immunologie et cancérologie,

Département de pathologie et biologie cellulaire, Université de Montréal, CP 6128, succursale centre-ville,

lisons principalement sur les données obtenues grâce aux modèles de la drosophile (Drosophila melanogaster) et du nématode (Caenorhabditis Montréal, Québec H3C 3J7 Canada.

marc.therrien@umontreal.ca elegans) qui ont contribué à l'élucidation de ce processus. Quoique les approches biochimiques aient résolu plusieurs aspects de cette question [2], l'exploitation de la génétique de la drosophile et du nématode au cours des années 1990 s'est avérée particulièrement utile, non seulement pour confirmer l'identité et l'ordre respectif des composantes de base de la voie de signalisation, mais également pour distinguer de nouveaux régulateurs (Tableau I).

\section{Premières descriptions de l'axe de signalisation RTK/RAS/ERK}

Les premières études génétiques de la signalisation RTK/RAS parurent en 1990. Le duo Min Han et Paul Sternberg, ainsi que le groupe de Robert Horvitz, ont isolé indépendamment RAS (LET-60) comme facteur positif agissant en aval du RTK EGFR (LET-23). Les deux groupes ont employé des techniques de génétique fondées sur la perturbation du développement de la vulve de $C$. elegans, un processus dépendant de la signalisation RTK [3]. Quelques mois plus tard, à l'aide d'une stratégie génétique analogue, mais exploitant le développement de l'œil de la drosophile, le groupe de Gerald Rubin décela RAS comme facteur requis en aval du RTK Sevenless (SEV, protein enhancer of sevenless 2B) [4]. Ensemble, ces travaux confirmèrent in vivo la position relative de RAS par rapport aux RTK, telle qu'on l'avait précé- 


\begin{tabular}{|c|c|c|c|c|}
\hline $\begin{array}{l}\text { Nom } \\
\text { générique }\end{array}$ & D. melanogaster & C. elegans & H. sapiens & Fonction moléculaire \\
\hline $14-3-3$ & $14-3-3 \varepsilon, 14-3-3 \zeta$ & FTT-2 (?), PAR-5 (?) & $\begin{array}{l}\text { YWHAE, YWHAB, YWHAZ, YWHAH, YWHAQ, } \\
\text { SFN(?), YWHAG }\end{array}$ & Adaptateur / chaperonne \\
\hline CBL & CBL & $\mathrm{SLI}-1^{*}$ & $\mathrm{CBL}, \mathrm{CBLB}, \mathrm{CBLC}$ & Ubiquitine ligase $\varepsilon 3$ \\
\hline CDC37 & $\operatorname{CDC} 37$ & CDC-37 (?) & CDC37, CDC37L1 (?), CDC37P1 (?) & Chaperonne \\
\hline CNK & CNK* & CNK-1 & CNKSR1, CNKSR2, CNKSR3 (?), IPCEF1 (?) & Adaptateur \\
\hline EIF4AIII & EIF4AIII* & Y65B4A.6 (?), F33D11.10 (?) & EIF4A3 (?) & Facteur d'épissage \\
\hline ERK & RL & MPK-1 & MAPK1 (દRK2), MAPK3 (દRK1) & $\mathrm{S} / \mathrm{T}$ kinase \\
\hline GAB & DOS & SOC-1 & $\mathrm{GAB} 1, \mathrm{GAB} 2, \mathrm{GAB} 3, \mathrm{GAB} 4$ (?) & Adaptateur \\
\hline GAP1 & $\mathrm{GAPl}$ * & GAP-1 & RASA2 (GAPIM), RASA3 & GAP \\
\hline GCKIII & GCKIII* & GCK-1 (?) & STK24 & $\mathrm{S} / \mathrm{T}$ kinase \\
\hline GRB2 & DRK & SEM- 5 & GRB2 & Adaptateur \\
\hline HSP9O & HSP83 & DAF-2l (?) & HSP90ABl, HSP90AAl (?) & Chaperonne \\
\hline нур & AVE (HYP)* & AVE-1 (?) & SAMD10 (?), SAMD12 (?) & Adaptateur \\
\hline KSR & $\mathrm{KSR}^{\star}$ & KSR-1*, KSR-2 & $\mathrm{KSR} 1, \mathrm{KSR} 2$ & Adaptateur \\
\hline MAGO & MAGO* & MAG-1 (?) & MAGOH (?), MAGOHB (?) & Facteur d'épissage \\
\hline MєK & DSORI & MEK-2 & MAP2K1, MAP2K2 & $\mathrm{S} / \mathrm{T} / \mathrm{Y}$ kinase \\
\hline MKP3 & MKP3 & LIP-1 & DUSP6, DUSP7, DUSP9 & S/T/Y phosphatase \\
\hline NF1 & NFl & - & NFl & GAP \\
\hline PP1 & $\begin{array}{l}\text { PP1-13C (?), PP1-87B } \\
(?), \text { PPl } \alpha-96 \mathrm{~A}(?)\end{array}$ & $\begin{array}{l}\text { GSP-1 (?), GSP-2 (?), GSP- } \\
3(?), \mathrm{PPH}-1 \text { (?) }\end{array}$ & PPPICA & $\mathrm{S} / \mathrm{T}$ phosphatase \\
\hline PP2A C & MTS & LहT-92 & PPP2CA, РPP2CB & $\mathrm{S} / \mathrm{T}$ phosphatase \\
\hline PP2A B & TWS (?) & SUR-6 & PPP2R2A, PPP2R2B, PPP2R2C, PPP2R2D & $\mathrm{S} / \mathrm{T}$ phosphatase \\
\hline PP2C & $\mathrm{ALPH} \star$ & TAG-93(?) & PPMIA, PPMIB (?) & $\mathrm{S} / \mathrm{T}$ phosphatase \\
\hline PPM1 & PPMI* & T23F1l.1(?) & PPMIA, PPMIB (?) & $\mathrm{S} / \mathrm{T}$ phosphatase \\
\hline PTP-ER & PTP-ER & - & PTPRR (PTP-SL), PTPN5 (STEP) & Y phosphatase \\
\hline PUM & PUM (?) & FBF-1*, FBF-2* & PUMI (?), PUM2* & Facteur liant I'ARNm \\
\hline RAF & $\mathrm{PHL}$ & LIN -45 & RAFl, BRAF, ARAF & $\mathrm{S} / \mathrm{T}$ kinase \\
\hline RAS & RAS85D & LET-60 & KRAS, NRAS, HRAS & GTPase \\
\hline RASGAP & VAP & GAP-2 & RASAl (pl20GAP) & GAP \\
\hline RNPS1 & RNPS1* & RNP-5 (?) & RNPSI (?) & Facteur d'épissage \\
\hline SHP-2 & CSW* & PTP-2 & PTPN11 (SHP2) & Y phosphatase \\
\hline SOS & SOS* & SOS-1 (LET-341) & SOS1, SOS2 & G\&F \\
\hline SPRY & STY* & - & SPRY1, SPRY2, SPRY3, SPRY4 & Adaptateur \\
\hline SRC & SRC42A & SRC-2 (?) & SRC, FRK & $y$ kinase \\
\hline SUR-8 & SUR-8 (?) & SOC-2 $(S \cup R-8) *$ & SHOC2 & Adaptateur \\
\hline TSU & TSU* & RNP-4 (?) & RBM8A (?) & Facteur d'épissage \\
\hline
\end{tabular}

Tableau I. Composantes de l'axe RTK/RAS/ERK communes à la drosophile, au nématode et à l'humain. Les symboles génériques représentés ici sont utilisés dans l'article pour désigner les homologues des protéines à travers les espèces. Les symboles officiels correspondants pour les protéines de D. melanogaster (FlyBase), C. elegans (WormBase) et les gènes de H. sapiens (HGNC) sont listés ici. Les symboles alternatifs communément employés sont entre parenthèses. Les homologues ne se trouvant pas dans la littérature ont été identifiées à l'aide de l'outil HCOP du HGCN (www.genenames.org), Ensembl (www.ensembl.org) ou par recherche BLAST. Dans un but de simplicité, les homologues des RTK ne sont pas présentés ici. Les astérisques indiquent les protéines qui furent initialement associées à la signalisation RTK/RAS/ERK grâce à la drosophile ou au nématode. Un point d'interrogation est placé à la suite des homologues pour lesquels une fonction dans la voie MAPK n'a pas été décrite. 
demment anticipée par des études biochimiques. II devenait donc clair que les mécanismes de signalisation induits par les RTK et RAS étaient apparentés et que l'application concertée d'approches génétiques et biochimiques permettrait vraisemblablement leur élucidation. En accord avec cette idée, plusieurs travaux effectués au cours de cette période permirent dans un premier temps de comprendre comment les RTK modulent l'activité de RAS. Par exemple, une étude réalisée chez la drosophile distingua SOS (son of sevenless) comme facteur essentiel à la signalisation RTK et le positionna entre les RTK et RAS [4]. Son homologie à CDC25 de levure, un facteur d'échange GDP/GTP pour RAS, suggéra que SOS joue probablement un rôle dans l'activation de RAS en favorisant sa liaison au GTP. Par ailleurs, le groupe de Robert Horvitz identifia chez C. elegans SEM-5 (sex muscle abnormal protein 5), un multi-adaptateur apparenté à GRB2 (growth factor receptor-bound protein 2) agissant aussi entre les RTK et RAS [3]. À la suite de ces découvertes, huit articles [5] publiés en 1993 ont montré comment les RTK activés recrutent SOS à leur portion cytoplasmique via GRB2, permettant ainsi l'activation de RAS. L'inactivation de RAS, quant à elle, avait été établie: elle est biochimiquement sous l'emprise des protéines GAP (GTPase activating proteins), deux protéines étant associées à cette activité, RASGAP et le suppresseur de tumeurs NFl [6]. Un criblage génétique chez la drosophile en identifia une troisième, GAPl [4].

Mais alors, qu'en était-il des trois kinases de base du module RAS/ ERK, soit RAF, MEK et ERK ? Dans les années 1980, il avait été montré que la transformation cellulaire par une forme oncogénique de RAF

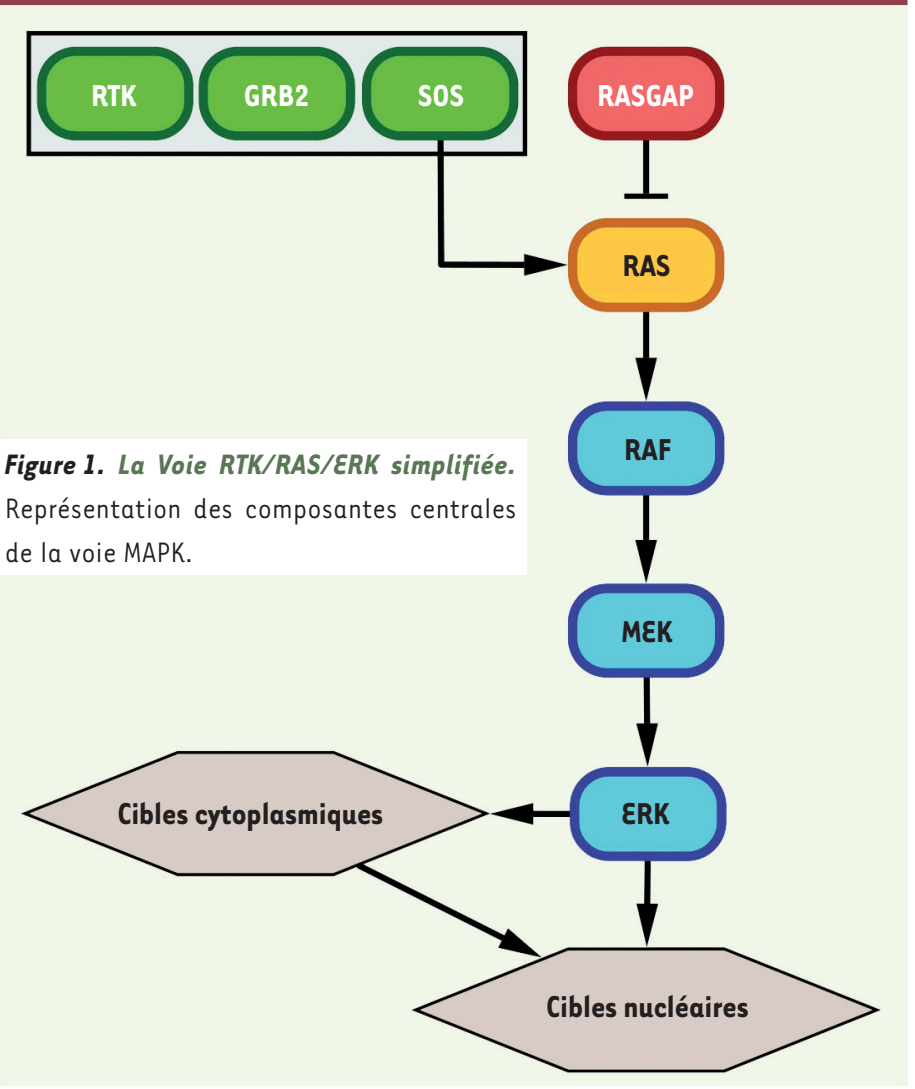

ne dépendait pas de RAS, mais qu'en revanche celle induite par RAS nécessitait RAF [2]. En accord avec ces résultats, Dickson et al. positionnèrent génétiquement RAF de la drosophile en aval de SEV, SOS et RAS [4], ce qui fut également confirmé chez C. elegans [3]. Quelque temps après, le groupe de Rubin identifia un complexe de protéines chaperonnes, HSP90 (HSP83) et CDC37, comme facteurs agissant en aval des RTK [7]. Leur rôle demeura incertain jusqu'à ce qu'une étude génétique chez la drosophile démontre leur importance pour l'activité de RAF [8]. Par la suite, des études biochimiques confirmèrent ces résultats en montrant que ces protéines sont essentielles pour le repliement et la stabilité de $\operatorname{RAF}[9,10]$. Le repérage de MEK par approche génétique fut réalisé chez la drosophile quelques mois après son identification biochimique. En effet, un criblage génétique basé sur la réversion de la létalité causée par un allèle mutant de RAF, permit l'identification de MEK comme facteur agissant en aval de RAF et des RTK [4]. Finalement, દRK fut positionnée par analyse génétique chez la drosophile et le nématode en aval de RAS et de RAF, mais en amont de facteurs de transcription RTK/RAS-dépendants [3, 4]. En l'espace de quelques années, les composantes centrales de la voie avaient donc déjà été identifiées et disposées dans l'ordre où nous les voyons maintenant représentées dans tous les documents de référence (Figure 1).

\section{Apport des modèles génétiques}

\section{Identification des modulateurs en aval des RTK}

Les approches génétiques se révélèrent aussi très utiles pour repérer de nouveaux éléments encore insoupçonnés. Par exemple, le groupe de Norbert Perrimon distingua une composante à action positive en aval du RTK Torso (TOR), nommée CSW (corkscrew; aussi appelée SHP-2), une tyrosine phosphatase comprenant deux domaines SH2 (src homology-2) [11]. La fonction de SHP-2 dans la voie RAS/ERK demeura nébuleuse jusqu'à l'identification d'une autre protéine agissant entre les RTK et RAS, nommée DOS (daugther of sevenless), homologue des GAB (GRB2-associated binding protein) de mammifères, une protéine à domaine $\mathrm{PH}$ (pleckstrin homology) [12]. En effet, il fut démontré que cette $\mathrm{GAB}$ recrute SHP-2 à la membrane plasmique après l'activation d'un RTK [13] et que cet événement permet une activation prolongée de RAS en déphosphorylant un site de liaison de RASGAP sur le RTK et probablement en favorisant le recrutement de GRB2 [14]. Parallèlement aux protéines GAP, de nouvelles protéines ayant un rôle négatif furent aussi repérées. Par exemple, une étude cherchant à découvrir des facteurs participant 


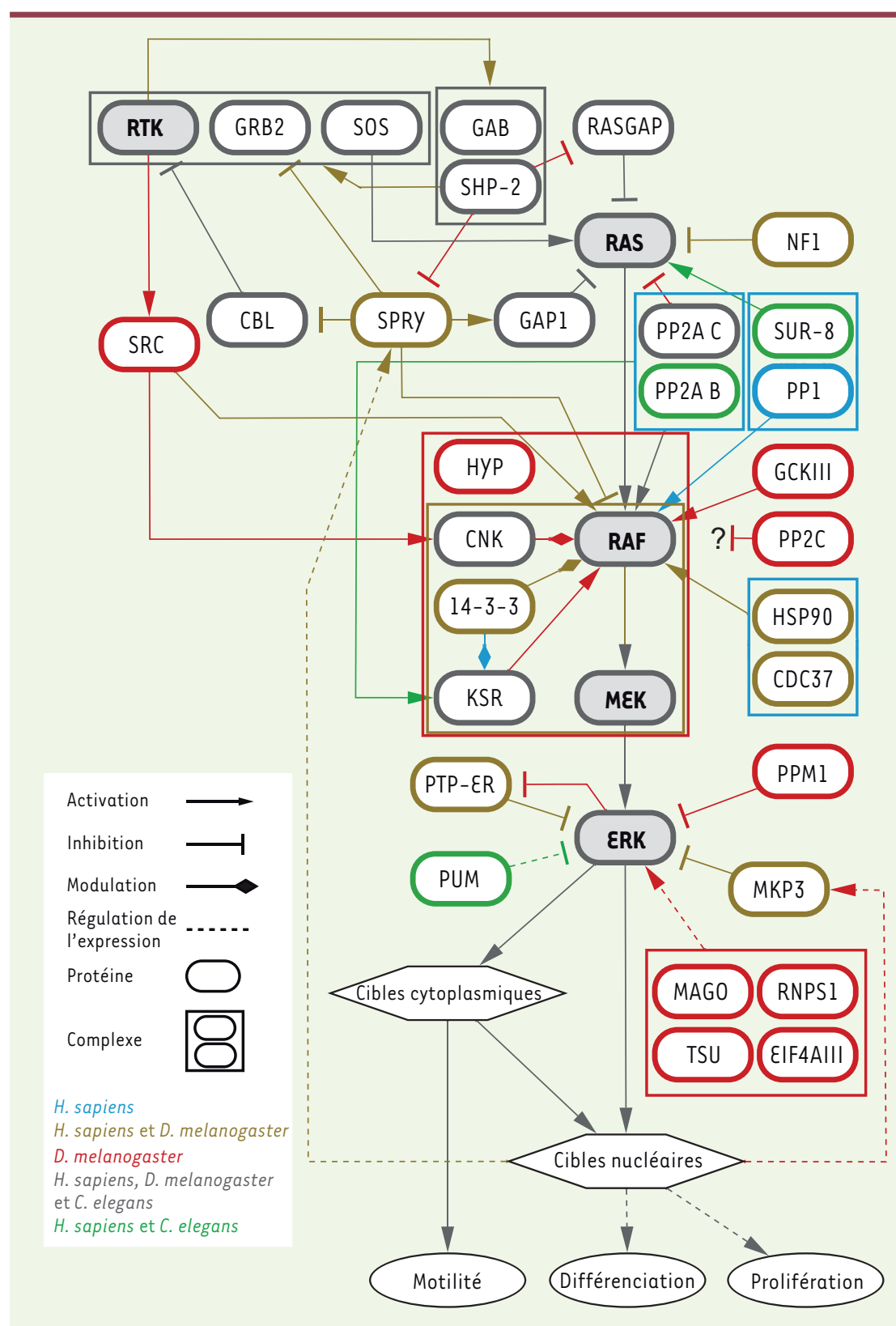

Figure 2. L'axe de signalisation RTK/RAS/ERK. Représentation du module RAS/ERK et des principaux régulateurs identifiés au moyen de techniques génétiques. Les composantes, modulation et complexes sont colorés selon la présence d'évidences chez $D$. melanogaster, C. elegans et/ou H. sapiens.

RAS/ERK [17]. Une autre composante à action négative est le proto-oncogène $\mathrm{CBL}$, une ubiquitine ligase de type $\varepsilon 3$ qui agit dans l'internalisation et la dégradation des RTK [18]. Cette dernière fut reliée pour la première fois à la signalisation RTK/RAS grâce à une étude génétique du développement de la vulve chez C. elegans [19]. De façon surprenante, il semblerait que SPRY ait également la capacité de lier et de séquestrer CBL. Cette fonction positive de SPRY sur la signalisation RTK lui confère donc un rôle plus nuancé qui pourrait dépendre du contexte cellulaire [16] (Figure 2).

Au milieu des années 1990, il avait été démontré que l'activation de RAS entraîne le recrutement et l'activation de RAF à la membrane cellulaire, provoquant ensuite la phosphorylation et l'activation en cascade de MEK et ERK [2, 20]. ERK activée phosphoryle à son tour un répertoire spécifique de substrats permettant ainsi une réponse cellulaire [20]. Quoique cette description simple de la voie RAS/ERK corresponde toujours à notre compréhension de celle-ci, plusieurs observations laissaient présager l'existence d'autres

au développement du système respiratoire de l'embryon de mouche identifia SPRY (sprouty) comme antagoniste de la signalisation ERK déclenchée par le FGF (fibroblast growth factor). L'étude démontra également que l'expression de SPRY est dépendante de la signalisation FGF, décrivant ainsi une boucle de rétroaction négative [15]. L'activité de SPRY n'est pas restreinte à la signalisation par le FGF, car elle module aussi d'autres RTK. Toutefois, son mécanisme d'action ne demeure que partiellement compris et différents scénarios ont été suggérés. Par exemple, selon le contexte cellulaire, SPRY semble capable d'inhiber l'activation de RAF, de séquestrer GRB2 ou bien de recruter GAPl au RTK [16]. De plus, des travaux récents ont montré que SHP-2 entre en jeu dans la déphosphorylation d'un résidu phosphotyrosine de SPRY nécessaire pour ses effets inhibiteurs. Ceci suggère donc un autre mode par lequel le complexe GAB/SHP-2 modulerait la signalisation protéines nécessaires à son fonctionnement. En 1995, les groupes de Rubin, Horvitz et Han effectuèrent des criblages génétiques dépendants de formes constitutivement actives de RAS chez la drosophile et le nématode dans le but de détecter de nouvelles protéines agissant en aval de RAS. Ces travaux permirent la découverte d'une nouvelle composante essentielle au module RAS/ERK, nommée KSR (kinase suppressor of RAS) [21-23]. Étant donné son étroite homologie avec RAF, on lui attribua d'abord un rôle de kinase agissant sur RAF ou dans une voie parallèle. Par contre, des études biochimiques et fonctionnelles suggérèrent que KSR est dépourvue d'activité catalytique et lui attribuèrent plutôt un rôle de protéine d'échafaudage 
capable d'une part, de recruter MEK à RAF et, d'autre part, de participer au processus d'activation de RAF par RAS [24]. Des études sur des souris chez lesquelles le gène KSRI fut inactivé ont confirmé son rôle dans la signalisation RAS/MAPK et ont montré que l'inactivation du gène provoque une perturbation du développement de tumeurs mammaires ou de papillomes RAS dépendants [25, 26]. De plus, KSRI et KSR2 semblent également avoir la capacité d'agir comme adaptateurs liant RAF, MEK et $\varepsilon R K[26,27]$. II convient de noter cependant qu'il existe très peu de données fonctionnelles sur KSR2. Finalement, l'inactivité de KSR en tant que catalyseur protéine kinase demeure toutefois contestée par certains [27].

\section{Régulateurs et protéines}

\section{constitutives des complexes de signalisation}

À la suite de la découverte de KSR, un criblage génétique basé sur un phénotype produit par l'expression d'une forme dominante-négative de KSR dans l'œil de mouche détecta CNK (connector enhancer of $K S R$ ) [28], une autre protéine multi-adaptatrice qui agit en reliant le complexe KSR/MEK et RAF. Des travaux récents de notre laboratoire ont montré que l'activité de CNK est régie par la tyrosine kinase Src42A (famille des kinases Src) [29]. Quoique le recrutement de RAF à CNK semble direct, celui de KSR/MEK à CNK fait intervenir une autre protéine appelée HYP (Hyphen ou AVE, Aveugle) [24]. Cette dernière n'est constituée que d'un domaine SAM (sterile alpha-motif) qui hétérodimérise avec le domaine SAM de CNK [30]. La formation d'un complexe comprenant RAF, KSR, MEK, CNK et HYP s'avère donc critique pour la transmission de signaux au sein du module RAS/ERK chez la drosophile. Non seulement la formation d'un tel complexe permet le recrutement de MEK à RAF, mais il participe directement au mécanisme d'activation de RAF où KSR joue un rôle essentiel indépendamment de sa propriété de protéine d'échafaudage [24]. En effet, la formation du dimère KSR-RAF induit l'adoption d'une conformation active par $\operatorname{RAF}[31,32]$. Chez les mammifères, deux des trois homologues de CNK, CNK1 et CNK2, interagissent également avec RAF et jouent un rôle dans la signalisation MAPK. Toutefois, l'importance du rôle de CNK1 et CNK2 semble varier selon le RTK stimulant la voie [27]. Pour ce qui est des deux homologues de HYP chez les mammifères, SAMD10 (SAM domain containing 10) et SAMD12, aucune étude n'est actuellement disponible à leur sujet.

Avec KSR et CNK, une autre protéine d'échafaudage, SUR-8, a été détectée chez C. elegans [33, 34]. L’homologue humain de SUR-8 aurait la capacité d'associer RAS et RAF [35]. En outre, une étude récente a montré que SUR-8 humain s'associerait plutôt à M-Ras, un membre distant de la famille Ras, de même qu'à la phosphatase PPl et que cet événement provoquerait la déphosphorylation d'un site de liaison des protéines 14-3-3 sur la partie amino-terminale de RAF [36]. Étant donné que l'association 14-3-3/RAFamino-terminal inhibe l'activité de RAF (voir ci-dessous), SUR-8 contribuerait à la signalisation RAS/ERK en défavorisant cette interaction.

Le criblage RAS-dépendant qui révéla KSR chez la drosophile permit aussi l'identification de la sous-unité catalytique de la sérine/thréonine-phosphatase PP2A, appelée MTS (microtubule star) [37]. Celle-ci semble jouer à la fois un rôle négatif entre RAS et RAF et un rôle positif en aval de RAF. Quoique le lien entre PP2A et le module RAS/ERK chez la Drosophile n'ait pas été étudié plus en détail, il est fort probable que son rôle positif soit relié à la régulation de l'association entre les protéines 14-3-3 et leurs sites de liaison phosphodépendants situés dans la partie amino-terminale de RAF et KSR, tel qu'on l'a observé chez les mammifères [38]. En effet, cette interaction séquestrerait RAF et KSR dans le cytoplasme, les maintenant ainsi dans une conformation inactive. Cependant, les protéines 14-3-3 n'agissent pas uniquement comme antagonistes: leur liaison à l'extrémité carboxy-terminale de RAF est également essentielle pour l'activité catalytique de ce dernier [39]. $\varepsilon$ n accord avec ces rôles-clés, deux études génétiques chez la mouche ont validé l'importance fonctionnelle des protéines 14-3-3 comme régulateurs de l'activité de $\operatorname{RAF}[40,41]$. Par ailleurs, des analyses génétiques de la sous-unité catalytique (LET-92) et régulatrice $B$ (SUR-6) de PP2A chez le nématode ont aussi confirmé l'importance de cette activité phosphatase pour réguler RAF et KSR [42, 43].

Une autre phosphatase découverte grâce au criblage RAS-dépendant chez la drosophile est PTP-ER (protein tyrosine phosphatase-ERK/enhancer of RAS) [44]. Celle-ci est apparentée de façon éloignée aux tyrosine-phosphatases PTP-SL et STEP de mammifères. Tout comme ses distants homologues, PTP-ER inhibe ERK en déphosphorylant le résidu tyrosine du motif TEY situé dans sa boucle activatrice. PTP-ER est ellemême phosphorylée et inactivée par $E R K$, décrivant ainsi une boucle de rétroaction. Par ailleurs, MKP3, une phosphatase à double spécificité (sérine/thréonine et tyrosine) agissant aussi sur ERK, a été mise en évidence par homologie de séquence chez la mouche et le nématode $[45,46]$. Une étude chez la drosophile a montré que PTP-ER et MKP3 peuvent agir indépendamment l'une de l'autre, ou de manière concertée selon le contexte cellulaire [47]. Plus récemment, l'existence d'une boucle de rétroaction négative engageant l'expression ERK-dépendante de MKP3 a également été décrite [48]. Finalement, PP2C (ALPH), un membre de la famille des sérine/thréonine phosphatases de type PPM (Mg-dependent protein phosphatase), a été identifiée par notre groupe à la suite du criblage KSR-dépendant mentionné ci-dessus. PP2C semble agir négativement à une étape en aval ou en parallèle de RAS [49]. L'identité de son substrat demeure inconnue. Cependant, sa capacité à agir sur les kinases des voies JNK et p38 fonctionnellement apparentées à RAF, suggère qu'elle pourrait aussi agir sur RAF et/ou KSR [50]. 
Le contrôle de la signalisation n'opère pas uniquement au niveau posttraductionnel: plusieurs évidences indiquent un contrôle de l'expression de composantes centrales de la voie. Par exemple, il a été montré que le miARN let-7 module l'expression de RAS chez $C$. elegans et chez l'homme [51]. Également chez C. elegans, FBF-1 (Fas-binding factor 1) et FBF-2, des facteurs de la famille Pumilio liant l'ARN, ont été montrés comme régulant la stabilité de l'ARN messager de ERK [52]. Plus récemment, notre groupe ainsi que celui de Jessica Treisman ont aussi montré que l'épissage des pré-ARN messagers de ERK de drosophile est régulé par quatre composantes du complexe $\varepsilon J C$ (exon junction complex): MAG0, TSU, EIF4AIII et RNPS1 [53, 54]. Ainsi, le contrôle du niveau d'expression des composantes centrales de la voie, tels que RAS et $\varepsilon R K$, ajoute un autre niveau de régulation au réseau RAS/ERK.

\section{Perspectives}

Nous avons présenté ici les principales protéines connues chez la drosophile et le nématode qui agissent entre les RTK et ERK (Figure 2 et Tableau I). Des homologues chez les mammifères ont été trouvés pour chaque nouvelle composante détectée, ce qui suggère que leur fonction est restée conservée au cours de l'évolution des métazoaires. Même si la majorité des protéines directement en cause dans cette voie de signalisation ont probablement été trouvées, il est aussi probable qu'il en reste encore de nouvelles à découvrir, de même que de nombreux modulateurs périphériques. Par exemple, un criblage à grande échelle utilisant une banque d'ARN interférants sur des cellules de drosophile en culture a récemment débusqué plusieurs nouvelles protéines dont la déplétion modulait la signalisation RTK/RAS/ERK [55]. Quoique pour la plupart leur caractérisation reste à venir, deux d'entre elles, une kinase de la famille STE20 (GCKIII) et une sérine/ thréonine phosphatase de la famille PPM (PPMI), semblent respectivement capables de réguler l'activité de RAF et d'ERK. Cette étude ne s'est pas limitée à répertorier de nouveaux régulateurs directs, elle a montré en outre l'importance de mécanismes de rétroaction, de même que la capacité de voies de signalisation parallèles, comme les voies JNK et Akt, à influencer la signalisation RTK/RAS/ERK. De plus, cette étude suggère que diverses machineries cellulaires, notamment celle participant à la biogenèse des miARN ou encore celle régulant le cycle cellulaire, ont pareillement la propriété de régir la signalisation RTK/ RAS/ERK. II semble donc que le niveau précis d'activation d'ERK dans une cellule donnée ne dépende pas uniquement de l'activation de la voie proprement dite, mais également de l'intégration de plusieurs signaux en provenance de diverses sources. Un défi pour les prochaines années sera d'identifier ces sources et de déterminer à quelle étape chacune d'elle s'inscrit. Un autre défi de taille sera d'élucider au niveau moléculaire la nature des diverses boucles de rétroaction et comment ces dernières sont utilisées pour régler dynamiquement l'intensité et la durée du signal. Ainsi, dans la mesure où ces phénomènes sont conservés, la drosophile et le nématode devraient, plusieurs années encore, servir d'alliés puissants dans la quête d'une compréhension fine des mécanismes moléculaires régissant la signalisation RTK/RAS/ERK. $\diamond$

\section{SUMMARY}

The greater RTK/RAS/ERK

signalling pathway: how genetics

has helped piece together a signalling network

Cells respond to changes in their environment, to developmental cues and to pathogen aggression through the action of a complex network of proteins. These networks can be split into a multitude of signalling pathways that relay signals from the microenvironment to the cellular components involved in eliciting a specific response. Perturbations in these signalling processes are at the root of multiple pathologies, the most notable of these being cancer. The study of receptor tyrosine kinase (RTK) signalling led to the first description of a mechanism whereby an extracellular signal is transmitted to the nucleus to induce a transcriptional response. Genetic studies conducted in drosophila and nematodes have provided key elements to this puzzle. Here, we briefly discuss the poorly known contribution of these multicellular organisms to our understanding of what has become a prototype in cell signalling as well as to the more recent description of the complex network of regulators that is now known to govern RTK/RAS/ERK signalling. $\diamond$

\section{CONFLITS D'INTÉRÊTS}

Les auteurs déclarent n'avoir aucun conflit d'intérêts concernant les données publiées dans cet article.

\section{REMERCIEMENTS}

Nous remercions Caroline Baril, Gino Laberge et Martin Lefrançois pour leurs commentaires sur le manuscrit. DAB est récipiendaire d'une bourse d'études doctorales des IRSC. MT est récipiendaire d'une chaire de recherche du Canada en signalisation intracellulaire. Ce travail fut financé par des fonds de recherche des IRSC et de la SCC octroyés à MT.

\section{RÉFÉRENCES}

1. Roberts PJ, Der CJ. Targeting the Raf-MEK-ERK mitogen-activated protein kinase cascade for the treatment of cancer. Oncogene $2007 ; 26: 3291-310$.

2. Malumbres $M$, Barbacid M. RAS oncogenes: the first 30 years. Nat Rev Cancer $2003 ; 3: 459-65$.

3. Sternberg PW, Han M. Genetics of RAS signaling in C. elegans. Trends Genet $1998 ; 14: 466-72$.

4. Wassarman DA, Therrien M, Rubin GM. The Ras signaling pathway in Drosophila. Curr Opin Genet Dev $1995 ; 5: 44-50$.

5. McCormick F. Signal transduction. How receptors turn Ras on. Nature 1993; $363: 15-6$.

6. Bernards A. GAPs galore! A survey of putative Ras superfamily GTPase activating proteins in man and Drosophila. Biochim Biophys Acta 2003; $1603: 47-82$.

7. Cutforth T, Rubin GM. Mutations in Hsp 83 and cdc 37 impair signaling by the sevenless receptor tyrosine kinase in Drosophila. Cell $1994 ; 77: 1027-36$.

8. van der Straten A, Rommel C, Dickson B, Hafen $\varepsilon$. The heat shock protein 83 (Hsp83) is required for Raf-mediated signalling in Drosophila. EMBO J 1997 $16: 1961-9$. 
9. Schulte TW, Blagosklonny MV, Ingui C, Neckers L. Disruption of the Raf-1-Hsp90 molecular complex results in destabilization of Raf-1 and loss of Raf-1-Ras association. J Biol Chem 1995 ; 270 : 24585-8

10. Grammatikakis N, Lin JH, Grammatikakis A, et al. p50 (cdc37) acting in concert with $\mathrm{Hsp} 90$ is required for Raf-1 function. Mol Cell Biol $1999 ; 19$ : 1661-72.

11. Perkins LA, Larsen I, Perrimon N. Corkscrew encodes a putative protein tyrosine phosphatase that functions to transduce the terminal signal from the receptor tyrosine kinase torso. Cell 1992 ; $70: 225-36$.

12. Raabe T, Riesgo-Escovar J, Liu X, et al. DOS, a novel pleckstrin homology domain-containing protein required for signal transduction between sevenless and Ras 1 in Drosophila. Cell 1996; $85: 911-20$

13. Herbst R, Zhang X, Qin J, Simon MA. Recruitment of the protein tyrosine phosphatase CSW by DOS is an essential step during signaling by the sevenless receptor tyrosine kinase. EMBO J 1999; 18:6950-61.

14. Cleghon V, Feldmann P, Ghiglione $C$, et al. Opposing actions of CSW and RasGAP modulate the strength of Torso RTK signaling in the Drosophila terminal pathway. Mol Cell 1998; 2 : 719-27.

15. Hacohen N, Kramer S, Sutherland D, et al. Sprouty encodes a novel antagonist of FGF signaling that patterns apical branching of the Drosophila airways. Cell $1998 ; 92: 253-63$.

16. Kim HJ, Bar-Sagi D. Modulation of signalling by Sprouty: a developing story. Nat Rev Mol Cell Biol 2004 $5: 441-50$.

17. Jarvis LA, Toering SJ, Simon MA, et al. Sprouty proteins are in vivo targets of Corkscrew/SHP-2 tyrosine phosphatases. Development $2006 ; 133: 1133-42$

18. Dikic I, Schmidt MH. Malfunctions within the Cbl interactome uncouple receptor tyrosine kinases from destructive transport. Eur J Cell Biol 2007 ; 86 : 505-12.

19. Yoon CH, Lee J, Jongeward GD, Sternberg PW. Similarity of sli-1, a regulator of vulval development in C. elegans, to the mammalian proto-oncogene c-cbl. Science $1995 ; 269$ : 1102-5.

20. Campbell SL, Khosravi-Far R, Rossman KL, et al. Increasing complexity of Ras signaling. Oncogene $1998 ; 17: 1395-413$.

21. Sundaram M, Han M. The C. elegans ksr- 1 gene encodes a novel Raf-related kinase involved in Rasmediated signal transduction. Cell $1995 ; 83: 889-901$.

22. Therrien M, Chang HC, Solomon NM, et al. KSR, a novel protein kinase required for RAS signal transduction. Cell $1995 ; 83: 879-88$.

23. Kornfeld K, Hom DB, Horvitz HR. The ksr-l gene encodes a novel protein kinase involved in Rasmediated signaling in C. elegans. Cell $1995 ; 83: 903-13$.

24. Douziech M, Sahmi M, Laberge G, Therrien M. A KSR/CNK complex mediated by HYP, a novel SAM domain-containing protein, regulates RAS-dependent RAF activation in Drosophila. Genes Dev 2006 ; $20: 807-19$.

25. Lozano J, Xing R, Cai Z, et al. Deficiency of kinase suppressor of Rasl prevents oncogenic ras signaling in mice. Cancer Res $2003 ; 63: 4232-8$.

26. Nguyen A, Burack WR, Stock JL, et al. Kinase suppressor of Ras (KSR) is a scaffold which facilitates mitogen-activated protein kinase activation in vivo. Mol Cell Biol 2002; 22 : 3035-45.

27. Claperon A, Therrien M. KSR and CNK: two scaffolds regulating RAS-mediated RAF activation. Oncogene $2007 ; 26: 3143-58$

28. Therrien M, Wong AM, Rubin GM. CNK, a RAF-binding multidomain protein required for RAS signaling. Cell $1998 ; 95: 343-53$.

29. Laberge G, Douziech M, Therrien M. Src42 binding activity regulates Drosophila RAF by a novel CNKdependent derepression mechanism. Embo J 2005 ; 24 : 487-98.

30. Rajakulendran T, Sahmi M, Kurinov I, et al. CNK and HYP form a discrete dimer by their SAM domains to mediate RAF kinase signaling. Proc Natl Acad Sci USA 2008 ; 105 : 2836-41.

31. Rajakulendran T, Sahmi M, Lefrançois $M$, et al. A dimerization-dependent mechanism drives RAF catalytic activation. Nature $2009 ; 461: 542-5$.

32. Lavoie H, Therrien M. Mécanisme d'activation de l'oncogène BRAF : I'union fait la force. Med Sci (Paris) $2010 ; 26: 459-60$.

33. Sieburth DS, Sun Q, Han M. SUR-8, a conserved Ras-binding protein with leucine-rich repeats, positively regulates Ras-mediated signaling in C. elegans. Cell $1998 ; 94: 119-30$.

34. Selfors LM, Schutzman JL, Borland CZ, Stern MJ. Soc-2 encodes a leucine-rich repeat protein implicated in fibroblast growth factor receptor signaling. Proc Natl Acad Sci USA 1998 ; $95: 6903-8$.

35. Li W, Han M, Guan KL. The leucine-rich repeat protein SUR-8 enhances MAP kinase activation and forms a complex with Ras and Raf. Genes Dev $2000 ; 14: 895-900$.
36. Rodriguez-Viciana P, Oses-Prieto J, Burlingame A, et al. A phosphatase holoenzyme comprised of Shoc2/Sur8 and the catalytic subunit of PPl functions as an M-Ras effector to modulate Raf activity. Mol Cell $2006 ; 22: 217-30$.

37. Wassarman DA, Solomon NM, Chang HC, et al. Protein phosphatase $2 A$ positively and negatively regulates Rasl-mediated photoreceptor development in Drosophila. Genes Dev $1996 ; 10: 272-8$.

38. Dougherty MK, Morrison DK. Unlocking the code of 14-3-3. J Cell Sci 2004 ; $117: 1875-84$.

39. Tzivion G, Luo Z, Avruch J. A dimeric 14-3-3 protein is an essential cofactor for Raf kinase activity. Nature $1998 ; 394: 88-92$.

40. Kockel L, Vorbruggen $\mathrm{G}$, Jackle $\mathrm{H}$, et al. Requirement for Drosophila 14-3-3 zeta in Raf-dependent photoreceptor development. Genes Dev 1997 ; 11 : 1140-7.

41. Chang HC, Rubin GM. 14-3-3 epsilon positively regulates Ras-mediated signaling in Drosophila. Genes Dev $1997 ; 11: 1132-9$.

42. Sieburth DS, Sundaram M, Howard RM, Han M. A PP2A regulatory subunit positively regulates Ras-mediated signaling during Caenorhabditis elegans vulval induction. Genes Dev 1999; 13 : 2562-9.

43. Yoder JH, Chong H, Guan KL, Han M. Modulation of KSR activity in Caenorhabditis elegans by $\mathrm{Zn}$ ions, PAR-1 kinase and PP2A phosphatase. EMBO J 2004 ; 23 : 111-9.

44. Karim FD, Rubin GM. PTP- $\varepsilon R$, a novel tyrosine phosphatase, functions downstream of Ras 1 to downregulate MAP kinase during Drosophila eye development. Mol Cell $1999 ; 3: 741-50$.

45. Berset $T$, Hoier \&F, Battu $G$, et al. Notch inhibition of RAS signaling through MAP kinase phosphatase LIP-1 during C. elegans vulval development. Science 2001 ; 291: 1055-8.

46. Kim SH, Kwon HB, Kim YS, et al. Isolation and characterization of a Drosophila homologue of mitogen-activated protein kinase phosphatase- 3 which has a high substrate specificity towards extracellular-signal-regulated kinase. Biochem J $2002 ; 361: 143-51$.

47. Rintelen F, Hafen $\varepsilon$, Nairz K. The Drosophila dual-specificity ERK phosphatase DMKP3 cooperates with the ERK tyrosine phosphatase PTP-ER. Development 2003; $130: 3479-90$.

48. Gomez AR, Lopez-Varea A, Molnar C, et al. Conserved cross-interactions in Drosophila and Xenopus between Ras/MAPK signaling and the dual-specificity phosphatase MKP3. Dev Dyn 2005; 232 : 695-708.

49. Baril C, Therrien M. Alphabet, a Ser/Thr phosphatase of the protein phosphatase 2C family, negatively regulates RAS/MAPK signaling in Drosophila. Dev Biol 2006 $294: 232-45$.

50. Baril C, Sahmi M, Ashton-Beaucage D, et al. The PP2C alphabet is a negative regulator of stress-activated protein kinase signaling in Drosophila. Genetics $2009 ; 181: 567-79$.

51. Johnson SM, Grosshans H, Shingara J, et al. RAS is regulated by the let-7 microRNA family. Cell $2005 ; 120: 635-47$.

52. Lee MH, Hook B, Pan G, et al. Conserved regulation of MAP kinase expression by PUF RNA-binding proteins. PLoS Genet 2007 ; 3 : e233.

53. Ashton-Beaucage D, Udell CM, Lavoie $\mathrm{H}$, et al. The exon junction complex controls the splicing of MAPK and other long intron-containing transcripts in Drosophila. Cell $2010 ; 143: 251-62$.

54. Roignant Jy, Treisman JE. Exon junction complex subunits are required to splice Drosophila MAP kinase, a large heterochromatic gene. Cell 2010; $143: 238-50$.

55. Friedman A, Perrimon N. A functional RNAi screen for regulators of receptor tyrosine kinase and ERK signalling. Nature $2006 ; 444: 230-4$.

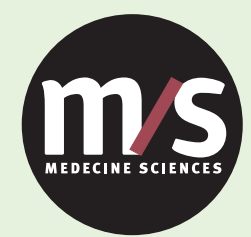

Tarifs d'abonnement M/S - 2010

Abonnez-vous

à Médecine/Sciences
$>$ Grâce à $m / s$, vivez en direct les progrès des sciences biologiques et médicales

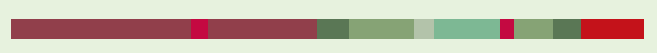

Bulletin d'abonnement page 1114 dans ce numéro de $\mathrm{m} / \mathrm{s}$
TIRÉS À PART

M. Therrien 